\title{
A Wide Range Temperature Sensor Using SOI Technology
}

\author{
R. L. Patterson ${ }^{\mathrm{a}}$, M. E. Elbuluk ${ }^{\mathrm{b}}$, and A. Hammoud ${ }^{\mathrm{c}}$ \\ ${ }^{\text {a }}$ Richard Patterson, NASA GRC, MS 309-2, Cleveland, OH 44135, USA \\ ${ }^{\mathrm{b}}$ Malik E. Elbuluk, ECE Dept., University of Akron, Akron, OH 44325, USA \\ c Ahmad Hammoud, ASRC Aerospace Corp., MS 309-2, Cleveland, OH 4413, USA
}

\begin{abstract}
Silicon-on-insulator (SOI) technology is becoming widely used in integrated circuit chips for its advantages over the conventional silicon counterpart. The decrease in leakage current combined with lower power consumption allows electronics to operate in a broader temperature range. This paper describes the performance of an SOIbased temperature sensor under extreme temperatures and thermal cycling. The sensor comprised of a temperature-to-frequency relaxation oscillator circuit utilizing an SOI precision timer chip. The circuit was evaluated under extreme temperature exposure and thermal cycling between $-190{ }^{\circ} \mathrm{C}$ and $+210{ }^{\circ} \mathrm{C}$. The results indicate that the sensor performed well over the entire test temperature range and it was able to re-start at extreme temperatures.
\end{abstract}

\section{Introduction}

Electronic systems in space exploration missions and in aerospace are expected to encounter extreme temperatures and wide thermal swings. Such missions include planetary surface exploration and deep space probes (hot and/or cold temperature), jet engine distributed control architecture (hot), and the NASA James Webb Space Telescope (cold). Electronics designed for such applications must, therefore, be able to withstand exposure to extreme temperatures and to perform properly for the duration of the missions.

Silicon-on-insulator (SOI) technology is becoming widely used in integrated circuit chips for its advantages over the conventional silicon counterpart (1). Reduced leakage current combined with lower power consumption allow the SOI-based electronics to function in a broader temperature operating range. In addition, SOI devices and circuits exhibit faster switching speeds and offer good tolerance to radiation. NASA could utilize this technology in the design of reliable standard as well as application-specific integrated circuits (ASIC) that are essential in space exploration missions and aerospace where severe operational conditions exist. Typically, SOI parts are designed for high temperature applications $\left(>200{ }^{\circ} \mathrm{C}\right)$ and improved radiation resistance. Very little information, however, exists about their performance at very low temperature conditions.

An effort was undertaken to design and develop a temperature sensor for distributed engine control architecture. Prompted by design considerations and ensuing benefits in terms of weight and cost reduction and improved reliability, the sensor needs to be located in the engine compartment, and it must be able to operate at temperatures exceeding $150{ }^{\circ} \mathrm{C}$. The output of the sensor, which consists of a stream of digitized pulses whose frequency is a function of the sensed temperature, would be interfaced with a controller or a computer. The data acquisition system would then give a direct readout 
of the temperature through the use of a look-up table, a built-in algorithm, or a mathematical model.

\section{Temperature Sensor Circuit}

The temperature-to-frequency relaxation oscillator circuit was constructed using a high temperature polyimide circuit board, Teflon wire interconnects, and high temperature lead-free solder. The circuit employed a high temperature SOI precision 555 timer. The timer is a ceramic-packaged chip and is specified for $-30{ }^{\circ} \mathrm{C}$ to $+225{ }^{\circ} \mathrm{C}$ operation (2). The other components of the circuit included a high temperature precision, thin film platinum RTD (Resistance Temperature Detector) as the temperature-sensing element, a solid tantalum filter capacitor, and two ceramic timing capacitors. A schematic of the circuit is shown in Figure 1, and a photograph of the circuit board is shown in Figure 2. The circuit was evaluated at selected test temperatures over the range of $-190{ }^{\circ} \mathrm{C}$ to $+210{ }^{\circ} \mathrm{C}$. The effect of thermal cycling was also investigated by subjecting it to a total of ten cycles within these temperature extremes using a rate of $10{ }^{\circ} \mathrm{C} / \mathrm{min}$ and a dwell time of ten minutes. The sensor circuit was evaluated in terms of its frequencyto-temperature conversion and variation in the output signal duty cycle and rise time with test temperature.

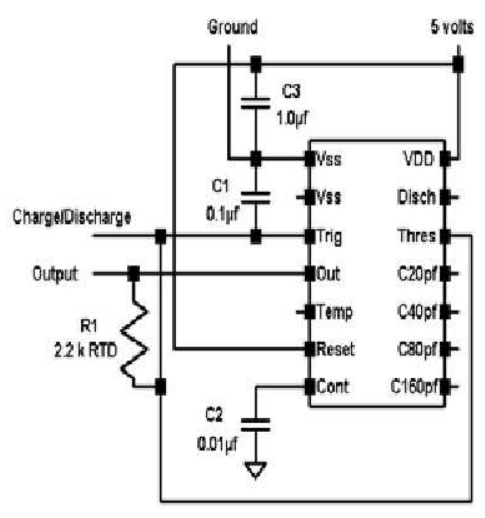

Figure 1. Circuit schematic.

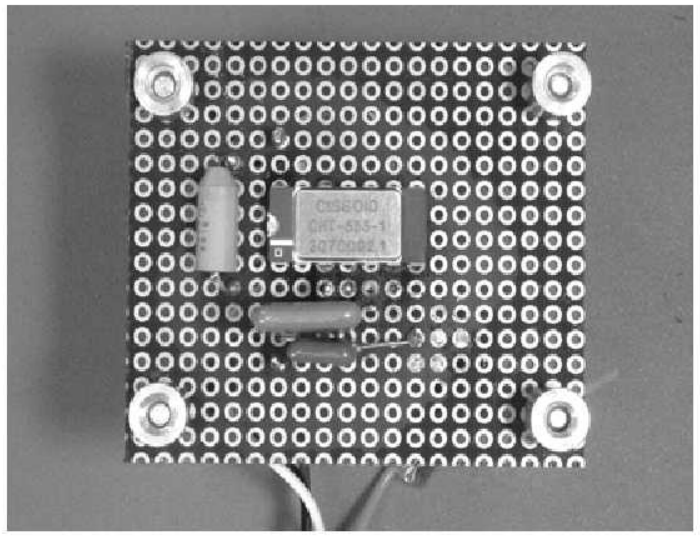

Figure 2. Sensor circuit proto-board.

\section{Test Results and Discussions}

The output response of the temperature-to-frequency conversion circuit, which comprised of a rectangular pulse train, is shown in Figure 3 at $25{ }^{\circ} \mathrm{C}$. The signal at the threshold pin, which governs the charge/discharge cycle of the timing capacitor $\mathrm{C} 1$, is also depicted in this figure as a triangular waveform. Those obtained at the high temperature of $+210^{\circ} \mathrm{C}$ and at the cryogenic temperature of $-190^{\circ} \mathrm{C}$ are shown in Figures 4 and 5, respectively. It can be clearly seen that the relaxation oscillator circuit performed very well throughout the temperature range between $+210{ }^{\circ} \mathrm{C}$ and $-190{ }^{\circ} \mathrm{C}$ as the output signal exhibited no distortion and its pulse count, i.e. frequency, fluctuated in response to variation in the sensed temperature. For example, while the frequency of the output signal had a value of about $3.393 \mathrm{kHz}$ at room temperature, it decreased to about $2.072 \mathrm{kHz}$ at $+210{ }^{\circ} \mathrm{C}$ and attained a magnitude of $15.048 \mathrm{kHz}$ when the temperature approached $-190{ }^{\circ} \mathrm{C}$. This frequency response, which took on a hyperbolic trend with 
temperature, is depicted in Figure 6. Plotting the period of the output signal versus temperature reveals, as expected, a near-linear response as shown in Figure 7. No change was experienced by either the duty cycle or the rise time of the output signal throughout the test temperature range as shown in Figures 8 and 9, respectively.

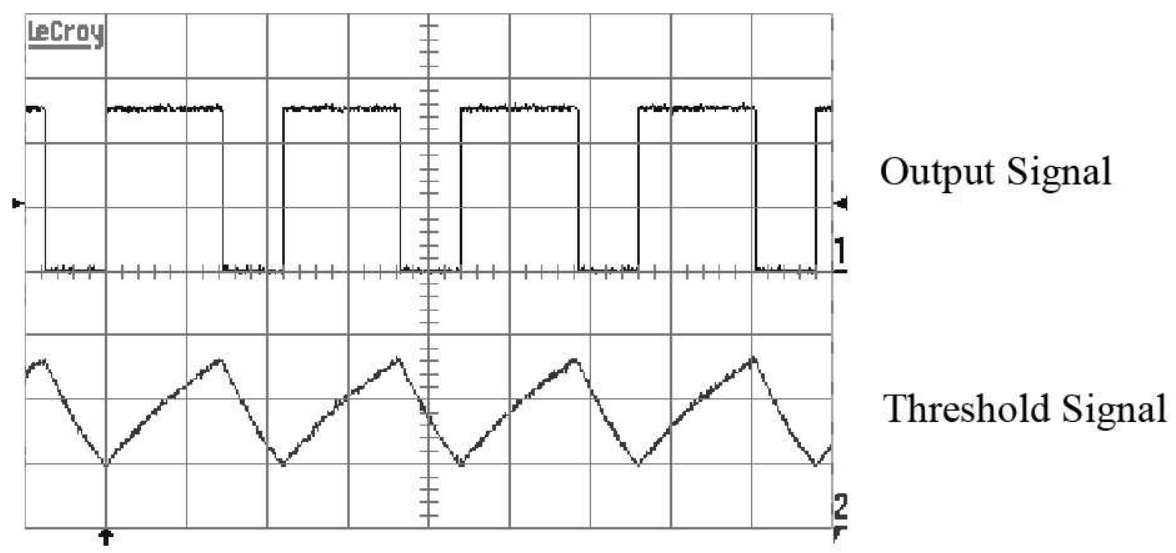

Figure 3. Output and threshold signal waveforms at $25^{\circ} \mathrm{C}$.

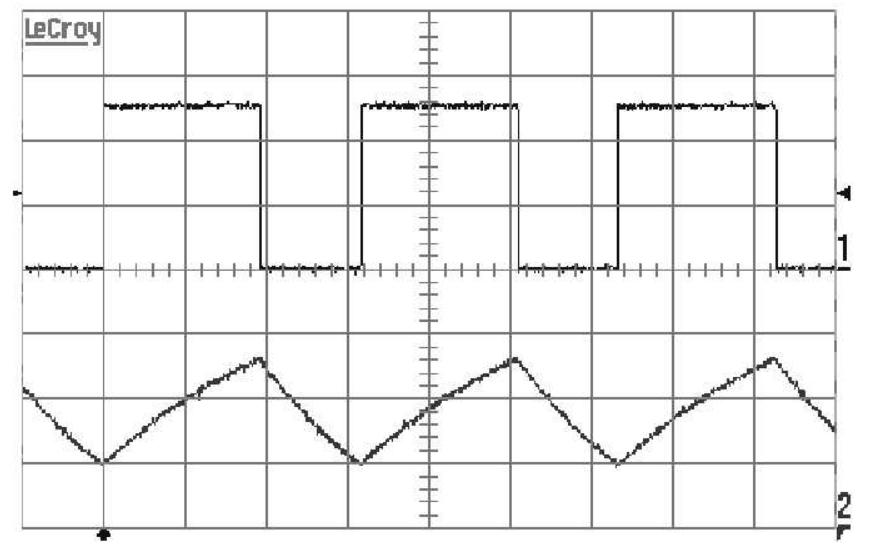

Output Signal

Threshold Signal

Figure 4. Output and threshold signal waveforms at $+210^{\circ} \mathrm{C}$.

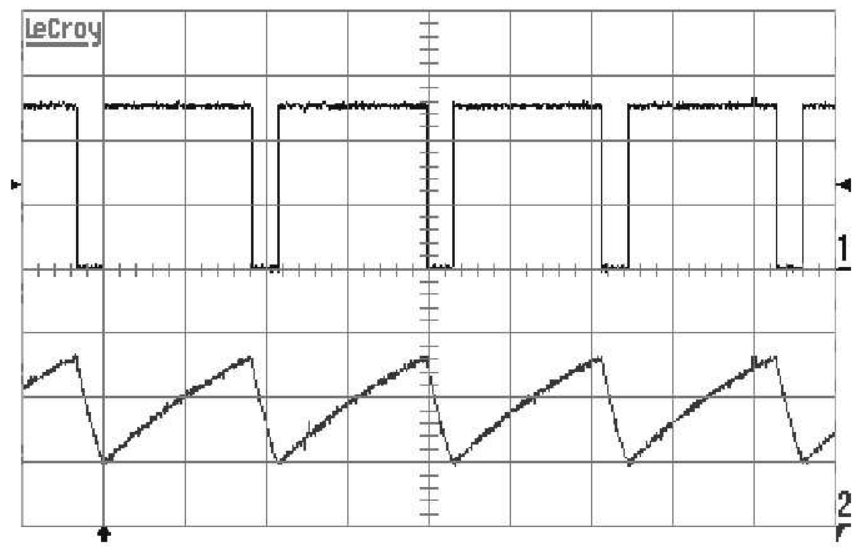

Output Signal

Threshold Signal

Figure 5. Output and threshold signal waveforms at $-190{ }^{\circ} \mathrm{C}$. 


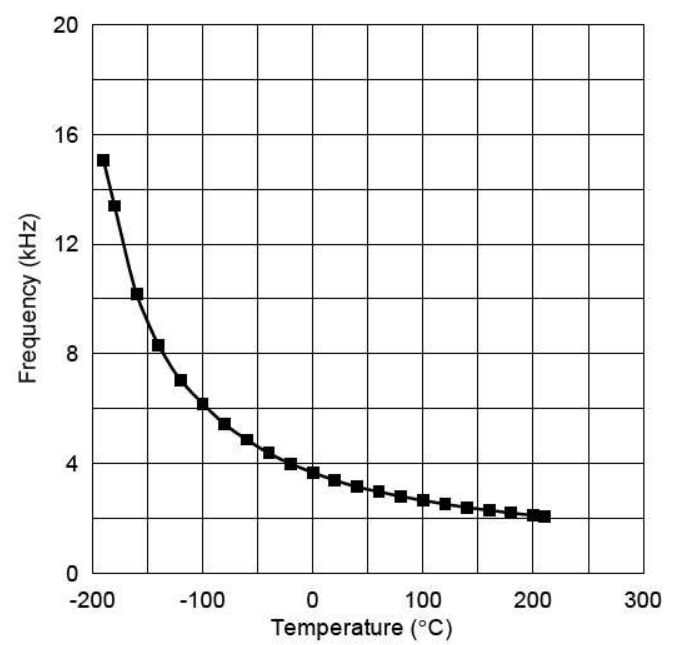

Figure 6. Frequency versus temperature.

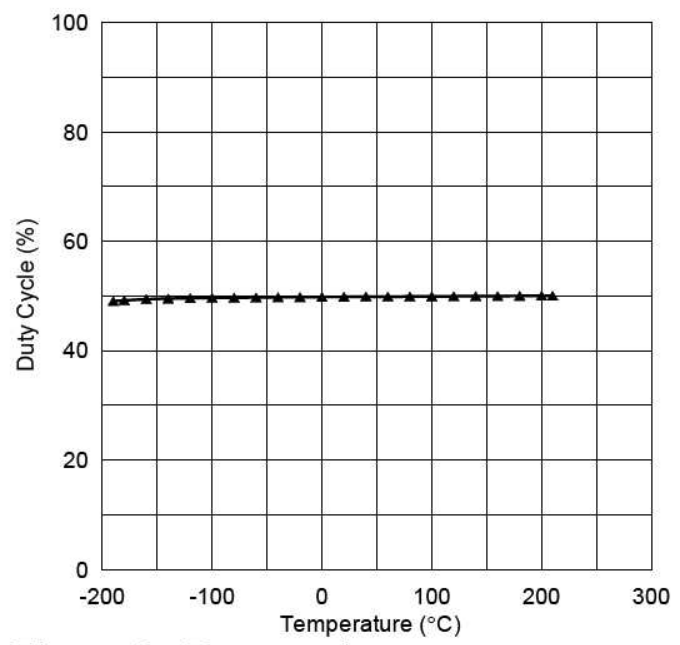

Figure 8. Duty cycle versus temperature.

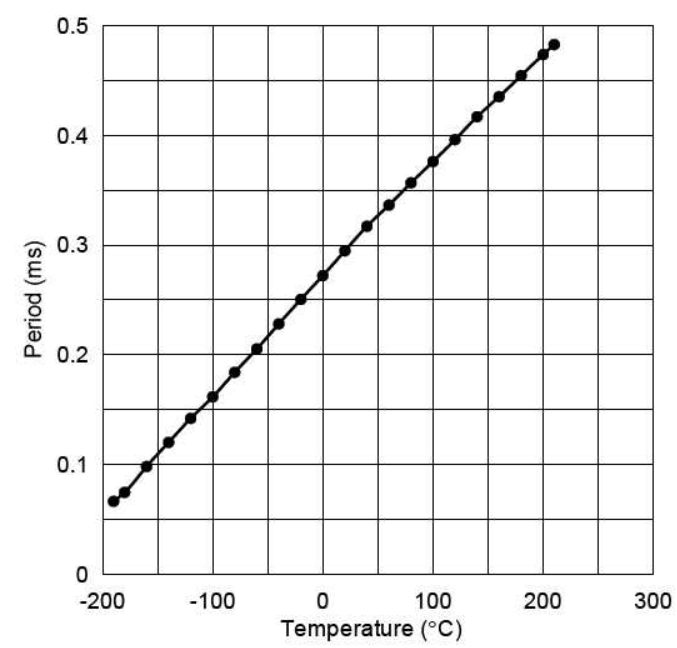

Figure 7. Period versus temperature.

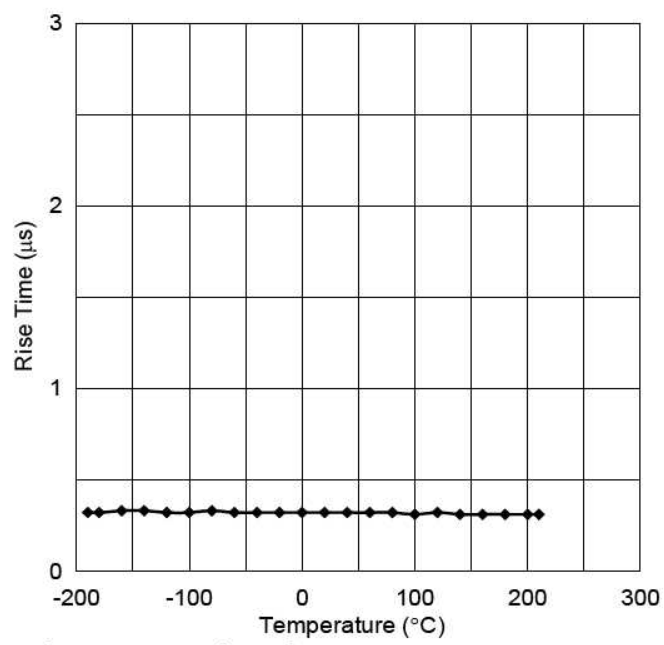

Figure 9. Rise time versus temperature.

The supply current of the circuit varied slightly with temperature as depicted in Figure 10. While the current hovered around $0.68 \mathrm{~mA}$ between $+20^{\circ} \mathrm{C}$ and $+210{ }^{\circ} \mathrm{C}$, it exhibited a slight increase with decreasing temperature, reaching about $2.5 \mathrm{~mA}$ at the extreme cryogenic temperature of $-190{ }^{\circ} \mathrm{C}$. This change in the circuit current with temperature is most likely attributed to the variation in the resistance value of the RTD element in the circuit as test temperature changed.

As was mentioned earlier, the performance of the relaxation oscillator circuit was also investigated after subjecting it to ten thermal cycles between $+210{ }^{\circ} \mathrm{C}$ and $-190{ }^{\circ} \mathrm{C}$. Postcycling measurements performed on the investigated parameters, i.e. frequency output, duty cycle, rise time, and supply current, revealed no major deviation from those obtained prior to cycling at any given test temperature. Therefore, this limited thermal cycling has had no impact on the operation of the oscillator circuit. In addition, the circuit demonstrated successful start-up operation while at the extreme temperatures, i.e. $+210{ }^{\circ} \mathrm{C}$ and $-190^{\circ} \mathrm{C}$. 


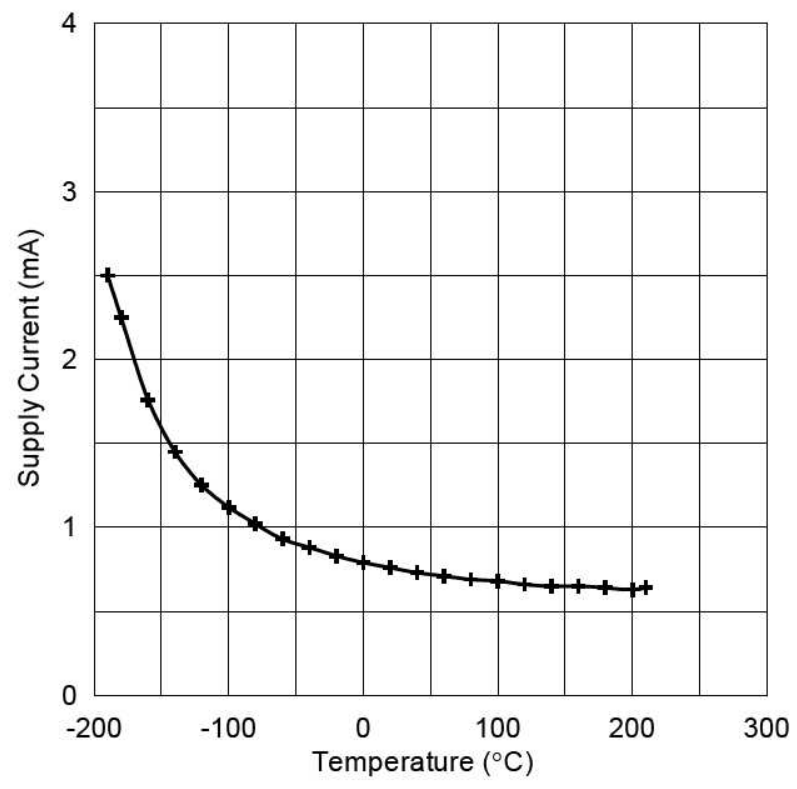

Figure. 10. Circuit supply current as a function of temperature.

\section{Conclusions}

A temperature-to-frequency relaxation oscillator circuit was constructed using COTS (Commercial-Off-The-Shelf) parts for application under extreme temperatures. The circuit employed a recently-developed high temperature silicon-on-insulator 555 timer, thin-film platinum RTD, and solid tantalum and ceramic capacitors. The circuit was designed mainly for hot jet engine environment, but it was evaluated also for potential use under cryogenic conditions. Performance of the oscillator circuit was investigated in terms of its temperature-sensing response, output signal duty cycle and rise time, and supply current under a wide temperature range between $-190{ }^{\circ} \mathrm{C}$ and $+210{ }^{\circ} \mathrm{C}$ and after thermal cycling. The prototype circuit performed well throughout this temperature range in producing a frequency output that was a function of the sensed temperature, and no major changes were observed in its characteristics, i.e. duty cycle and rise time of the output signal, as a result of change in test temperature. In addition, all of the individual parts exhibited no physical or packaging damage due to the extreme temperature exposure or cycling. These preliminary results suggest that the circuit has good potential for use in extreme, both hot and cold, temperature environments.

\section{Acknowledgments}

This work was performed under the NASA Glenn Research Center, GESS-2 Contract \# NNC06BA07B. Support was provided by the NASA GRC Fundamental Aeronautics / Subsonic Fixed Wing Program and by the NASA Electronic Parts and Packaging (NEPP) Program.

\section{References}

1. R. Patterson, A. Hammoud, and M. Elbuluk, Silicon-On-Insulator Operational Amplifier for Extreme Temperatures, NASA Report, $\underline{\text { http://www.nepp.nasa.gov. }}$

2. Cissoid Company, CHT 555 High Temperature Precision Timer, Datasheet Doc. DS07006 Rev. A, (March 28, 2007). 